\title{
Development of Investment Cases for Globally-Coordinated Management of Infectious Diseases
}

\author{
Kimberly M. Thompson ${ }^{1,2 *}$ and Radboud J. Duintjer Tebbens ${ }^{1}$ \\ ${ }^{1}$ Kid Risk, Inc., 10524 Moss Park Rd., Ste 204-364, Orlando, FL, 32832, USA \\ ${ }^{2}$ College of Medicine, University of Central Florida, Orlando, FL, USA
}

\begin{abstract}
Although developed countries tend to rapidly adopt and maintain commitments to interventions to manage infectious diseases, global adoption and maintenance varies significantly and depends on resources. Different national policies and preferences aggregate to the overall global path. The ability of infectious diseases to travel across borders makes countries interdependent with respect to managing their risks, and national, regional, and global health leaders demand information that can help to support global coordination, strategy, planning, and resource development and allocation decisions. The Global Vaccine Action Plan identifies the need to develop investment cases to support vaccine finance decisions. Building on prior generic discussions of global disease management investment case concepts, we developed an outline of specific attributes for inclusion in investment cases. We engaged motivated stakeholders in a process to evaluate the proposed content for inclusion in investment cases for global management of infectious diseases in a consultation process. This paper reports on the final proposed content for investment cases and the insights from the stakeholder consultation process.
\end{abstract}

Keywords: Infectious disease; Financing; Economic analysis; Immunization; Global coordination

\section{Introduction}

The Global Vaccine Action Plan (GVAP) promotes the vision of a world "in which all individuals and communities enjoy lives free from vaccine-preventable diseases" and it seeks to extend the full benefits of immunization to all people by 2020 and beyond [1]. The GVAP recognizes that achieving this vision will require significant investments, and indicates that as countries deal with difficult decisions related to competing health priorities: "expenditures must be linked to outputs and impacts, showing a clear investment case for immunization" [1]. Developing immunization investment cases has emerged as a new approach to ensuring sufficient commitment of resources and realizing the full benefits of vaccines.

Despite the demand for investment cases, little guidance currently exists. Economic analyses and dynamic disease modeling continue to play an increasingly important role in disease control and management by providing critical information for policy makers and stakeholders about the risks, costs, and benefits of their options and decisions, particularly as newer and more expensive vaccines and other interventions become available, the size of the global population increases, and commitments of resources to immunization grows [2]. The decision to control or eradicate a disease represents a choice that requires the investment of financial, human, and other resources, and given the abilities of diseases to spread across borders, countries face interdependent disease management choices and outcomes that necessitate coordination [3-5]. However, even a global commitment to eradication and coordination of activities may prove insufficient to achieve the goal. This may occur, at least in part, because decision makers may agree to the goal without a good understanding of the efforts required to achieve it (i.e., they agree in principle to the concept, but not necessarily to the commitment of the required resources and actions). For example, with respect to the 1988 World Health Assembly commitment to eradicate polio, "some delegates to the assembly in 1988 might not have made a truly informed decision on the launching of the initiative, since there had been no clear statement on resource requirements or strategies" [6]. The lack of a clear understanding of strategies and resource requirements most likely contributed to a slower intensification of vaccination than needed to meet the initial target date of eradication of all three serotypes of wild polioviruses (WPVs) by 2000, although the Global Polio Eradication Initiative (GPEI) achieved eradication of type 2 WPV by 2000 [7].

Global health donors and policy makers increasingly want to know what to expect and demand detailed information about potential challenges that could lead to delays and increase costs. Lessons learned from the GPEI experience motivate the development of tools to help stakeholders of potential globally-coordinated disease management initiatives make informed decisions about whether and how much to commit and how to manage expectations and ensure adequate financing to meet the commitments made [8-10]. While the concept of investment cases exists for some vaccine initiatives, for example in the context of decisions made by the GAVI Alliance related to the selection of vaccines for inclusion in its portfolio [11], such cases do not currently exist with respect to coordination of global disease management efforts. In 2005, the World Health Organization (WHO) and partners launched the Global Immunization Vision and Strategy (GIVS), 2006-2015 [12], which proposed the first ten-year strategic plan to globally expand the use of vaccines to prevent disease and led to development of a Global Vaccine Action Plan (GVAP) [1]. Economic analyses strongly support investments in vaccination overall while also highlighting the reality that immunization remains underused and undervalued globally [13-16], which further underscores the need for efforts to develop investment cases for vaccines [17]. Recent analyses promise significant health and

*Corresponding author: Kimberly M. Thompson, Kid Risk, Inc., 10524 Moss Park Rd., Ste 204-364, Orlando, FL, 32832, USA, Tel: 407-266-7037; E-mail: kimt@kidrisk.org

Received November 26, 2012; Accepted December 15, 2012; Published December 19, 2012

Citation: Thompson KM, Duintjer Tebbens RJD (2012) Development of Investment Cases for Globally-Coordinated Management of Infectious Diseases. J Vaccines Vaccin 3:164.doi:10.4172/2157-7560.1000164

Copyright: ( 2012 Thompson KM, et al. This is an open-access article distributed under the terms of the Creative Commons Attribution License, which permits unrestricted use, distribution, and reproduction in any medium, provided the original author and source are credited. 
economic benefits for efforts like the GIVS and GVAP [18,19] despite the large costs of scaling up immunization [20].

The extent of global coordination of efforts to manage infectious diseases varies by disease, ranging from extensive coordination by the GPEI [21], the Measles and Rubella Initiative [22], and the Maternal and Neonatal Tetanus Elimination Initiative [23] to essentially no global coordination for cholera or parasitic diseases [24]. Given the large portfolio of diseases to manage, we built on prior generic discussions $[9,10]$ to develop an initial list and descriptions of content for inclusion in investment cases that will provide synthesized information to support global policy and resource development and allocation efforts. We use the term "investment case" to cover all of the required investments by all stakeholders, including political, social, systemic, financial, and other resource commitments. In this regard, our concept of an investment case seeks to address the interests of multiple audiences representing a broad group of stakeholders, which differs from an investment case made solely for the consideration of financial resource commitments or prepared for the consideration by one particular audience (e.g., GAVI Alliance investment cases related to the allocation of its financial resources to support the introduction and accelerated adoption of new vaccines in its eligible countries) [11]. We emphasize that coordination of efforts does not necessarily imply the same activities in every country [25] and that countries make their own national immunization decisions, albeit with consideration of regional and global agreements and in the context of resource constraints [26-28].

The Measles and Rubella Initiative recently released a strategic plan for 2012-2020 [22] that charts a course for achieving global targets for measles and rubella set by the World Health Assembly (WHA) in May 2010 [29]. Although the WHA established these targets without the support of a clear investment case, ongoing discussions about potential eradication [30] and the recent resurgence of measles in Africa and Europe [31,32] suggest the need for synthesizing the information required to inform global decisions about the benefits and costs of current and future investments. Recognizing the heterogeneity in current commitments, disease burdens, and national and regional abilities to prevent and control measles and rubella, combined with the global trend toward commitments by regions toward eliminating measles and rubella [22], this represents an important time to explore the investment case for coordinated global management of measles and rubella. As a first step we identified the need to engage stakeholders in a process to develop the generic content for inclusion in investment cases. The next section describes the methods that we used to develop the content and identify and engage stakeholders to discuss the attribute list and descriptions. The following section provides our synthesized results for the content for investment cases, which we discuss in the last section.

\section{Methods}

We reviewed the literature for existing guidelines and recommendations related to the development of economic analyses and investment cases. We used the initial list of critical attributes for the development of a generic eradication investment case [9] and developed concise descriptions of the information covered by each item with consideration of the existing guidelines related to preparing economic analyses for immunization programs [33]. The content and process focus on creating investment cases that will facilitate engagement and rigorous evaluation by stakeholders as they consider moving from the status quo path (i.e., base path comparator) to some other option, with full consideration of what global commitments entail and while recognizing some uncertainty about the current path $[9,10]$. We took the objective of the process of developing an investment case as synthesizing all of the relevant information to characterize the: "global burden of disease; expected benefits, risks, and costs of the eradication and comparator options; social, political, and economic challenges; ethical considerations; and operational and other research needs" [9].

We shared the proposed draft investment case content with stakeholders identified through a simple process. Specifically, we posted the draft investment case on the web and emailed requests for input to 100 individuals representing 25 organizations nominated by partners of the Measles and Rubella Initiative. We also requested input from experts working on the development of investment cases related to several neglected tropical diseases. We presented the draft at two meetings during which we received verbal comments. Through an input form on the website (open between March 1, 2011 and April 6, 2011), we received written comments from 31 individuals representing 11 organizations. The form, which offered the option of responding anonymously, asked for stakeholder input on the following:

- What specific comments would you like to make about the text of the proposed outline and descriptions?

- What audience(s) do you believe the investment cases need to target?

- Do you believe that the proposed content will provide the information needed by the target audience(s)?

- If not, what do you believe is missing, needs adjustment, or requires clarification?

- With respect to measles and rubella, what thoughts would you like to share with us at this time about the current path and how we might characterize the expected status quo? What thoughts would you like to share with us at this time about any alternative paths and how we might characterize them? Please note the investment cases will include consideration of the eradication of measles and rubella separately and together, and we particularly invite your thoughts on any issues related to the achievement of existing regional and global targets and goals as well as your thoughts on timing in the context of the overall portfolio of global disease management efforts.

- What ideas, concerns, thoughts, experiences, data, information, or other topics would you like to share with us at this time, if any?

After collecting all of the comments from stakeholders, we iterated on the draft and developed the final list and description of attributes described in the next section.

\section{Results}

Over the course of the iterative process we determined the need to organize the required content for an investment case into five main sections: Context, Current situation, Alternatives, Considerations, and Conclusions followed by End Matter. We developed the list of attributes for the investment cases provided in $\mathrm{T}$ able 1, which includes descriptions of the information that each attribute should synthesize and present. We focused on developing the generic construct for an investment case, but our iteration and review process primarily engaged stakeholders focused on measles and rubella, and some neglected tropical diseases, and consequently this list may not capture all of the issues relevant to other diseases and/or types of interventions. We suggest that developers of other 


\section{Attribute} Description

\section{Context}

1.1 Objectives and scope

1.2 Description of the disease and its global health significance portfolio of coordinated global health priorities
.3 Role in the

Each investment case should begin with a concise introduction that provides critical context about the motivation and objective for the investment case. It should also clearly identify the scope and time horizon of the analysis and demonstrate the need for potential global coordination of the investment for the disease(s) considered (i.e., demonstrate the interdependencies that nations face with respect to disease management that necessitates the development of an investment case).

Each investment case should include a very brief description of the disease and existing and potential interventions for its management, and summarize the current state of any efforts to globally coordinate disease management. The discussion of global health significance should describe the extent to which heterogeneity exists with respect to current disease-related mortality and morbidity and disease management efforts, address issues related to the ability of the disease to spread regionally and globally, and identify important issues further developed in other sections of the investment case. If the investment case includes eradication as an option then it should present evidence of some demand for eradication and willingness to cooperate at the global level.

The investment case should briefly describe the status of the disease(s) considered in the investment case in the context of the overall portfolio

of coordinated global health priorities and discuss the degree of alignment of the investment case with any existing global vaccine strategic plans (e.g., Global Immunization Vision and Strategy (GIVS), 2006-2015; Global Vaccine Action Plan (GVAP)) [11,12]. This part should provide context about the collection of all similar investment cases and include a high-level discussion of current global health priorities (i.e., those with greatest global political and/or financial commitments or other internal and/or external investments of any type) in the context of the overall portfolio.

Any investment case will reflect the information available at the time of its preparation and the process used to prepare it. Consequently, this part of the investment case should summarize the process used to prepare it. Further, given the anticipated desire and/or expected need to maintain an investment case as a "living document" that evolves over time as conditions change, the discussion in this part should provide details about any important reasons for major changes (e.g., achievements of milestones, recognition of barriers and/or unanticipated events that lead to modifications of plans or expectations, etc.) The discussion should also summarize the lessons learned and iterations in the investment case over time and provide the reader with context related to the evolution of the investment case.

\section{Current path}

2.1 Mechanisms of disease transmission 2.2 Current treatments for the disease

2.3 Current global strategies for surveillance

The investment case should provide a description of the current state of knowledge about the nature of the disease and its transmission (including any host reservoirs). This should include discussion of the biological feasibility of eradication of the disease.

The investment case should provide details about the health effects caused by the disease and current standards of care with respect to treatment. This part should address any variability that currently exists with respect to treatment due to differences in the structure and utilization of health systems.

The investment case should provide a synthesis of the methods used for infection and/or disease surveillance and describe the current state and quality of surveillance evidence and context that might impact the interpretation of historical data provided by the surveillance system (e.g. historical development of methods, standards, and regional and/or global networks). For diseases considered eradicable biologically (discussed in 2.1), the investment case should discuss the technical feasibility of deploying surveillance activities sufficient to support globally-coordinated disease management efforts like those required to eradicate the disease and to confirm the absence of the pathogen with high confidence. This part should consider the need for sustainability of surveillance systems for the disease over time.

2.4 Existing interventions to prevent or stop transmission The investment case should provide a description of the current state of knowledge of the interventions that exist to protect humans from the disease and/or prevent its transmission, which should focus on synthesizing evidence of the effectiveness and practicality of the existing interventions. For diseases considered eradicable biologically (discussed in 2.1), the investment case should discuss the technical and programmatic feasibility of deploying the existing preventative interventions globally.

2.5 Current expected Recognizing that countries and regions may progress through various phases of exposure to the disease (e.g., emergence, endemic, epidemic) global level of control (i.e., the status quo) and control (i.e., none to eradication) [8,9] the investment case should summarize the current state of the world with respect to the geographic distribution of the disease and its control with characterization of the heterogeneity of disease burdens. The discussion should provide context about outbreaks and the extent of implementation of the existing interventions for prevention and in reaction to outbreaks (discussed in 2.4). This part should also synthesize the available data and the results of appropriate mathematical models used to define the status quo path, which reflects the past, current, and expected planned investments of countries with respect to control of the disease and represents the expected global baseline for comparison. Stakeholders may differ with respect to their perspectives on the current path (discussed in 2.5), and in this case the investment case should highlight underlying reasons for different perspectives and identify the need to recognize either more than one potential current path or different perspectives as alternative paths (discussed in section 3). Any regional and/or global minimum commitments agreed to by all countries will determine the minimal activities with respect to implementation of interventions, but individual countries can always exceed the minimum and the investment case should anticipate heterogeneity [27]. In the context of characterizing the current path, the investment case should synthesize the available historical data, model results, and other information about the disease burden up to the current time with consideration of any uncertainty related to the lack of perfect surveillance (discussed in 2.3). In addition, the investment case will need to forecast the expected future investments based on extrapolation from the past, current practices, and/or existing national, regional, and/or global commitments. Given the need to project into the future, the investment case may need to include more than one potential expected current or status quo path to account for uncertainty (e.g., unknown national and regional commitments to various interventions and/or inadequate evidence of secure financial support for existing commitments). The investment case must make any assumptions about projections transparent and demonstrate the impact of different potential projections with respect to the characterizations of the burden of disease, which may warrant elaboration in the context of uncertainty and/or sensitivity analyses (discussed in 2.9). The discussion should also highlight any major issues that currently interfere with greater success of the current path (e.g., refusal of an intervention by specific groups and/or in some geographic areas).

2.6 Characterization The investment case should synthesize the evidence about the financial costs of the expected current or status quo path(s). This should include of current financial costs projected, expected and realistic (i.e., not optimistic) costs of all of the interventions out over time, using a consistent currency year with all costs discounted back to the current time using a rate of $3 \%[1,32]$. The investment case should present appropriate break downs of the expected costs for regions and/or individual countries to the extent required to support the global analysis. The investment case should clearly state any assumptions related to projections of prices and utilization of the interventions consistent with the assumptions used to define the status quo path(s) (discussed in 2.5) and it should take care to factor in the impact of any time delays and unplanned events (i.e., include costs associated with the expectation of the need to implement contingency plans, discussed in 4.3). The investment case should reflect a societal perspective and characterize the direct costs of the interventions with quantification of indirect financial costs to the extent possible. It should also discuss different perspectives (e.g., health system, intervention manufacturer, individual) to highlight and discuss financial incentives faced by various stakeholders. The cost analysis should include the savings of health care treatment costs avoided due to the interventions, which implies that if the interventions themselves save money on net (i.e., if the interventions cost less than the amount of health care costs that society would spend to treat cases in the absence of the interventions), then the characterization of costs would imply net savings or a negative financial cost for the interventions. To the extent that the current path includes add-on or cost-sharing activities (e.g., giving vitamin A as part of polio or measles immunization campaigns, shared surveillance platforms), the analysis should separately quantify the costs of those activities and/or the impact of cost-sharing. 
2.7 Characterization of current health benefits
The investment case should synthesize the evidence about the health benefits of the current status quo path(s), building on the information in 2.5. This part should include discussion and use of different metrics that effectively address the needs of stakeholders to understand and communicate the health benefits of the current path (e.g., cases prevented, deaths prevented, disability-adjusted life years (DALYs) saved), and synthesize the available evidence related to economic valuation of the health benefits recognizing that countries may differ significantly with respect to their values $[8,9]$. This analysis should discuss assumptions about valuation of the health effects and present results in the context of the variability and uncertainty that exists about the values that countries place on health and it should attempt to capture the true net societal costs of the current path. To the extent that the current path includes add-on or cost-sharing activities (e.g., vitamin A delivery as part of polio or measles immunization campaigns), the analysis should separately quantify the health benefits of those activities and costs attributed to the interventions (discussed in 2.6).

2.8 Synthesis of the economics of the current investment 2.9 Limitations and uncertainties

3. Alternative paths

3.1 Alternative global options for control and/or eradication (including posteradication)

The investment case should synthesize the overall evidence and provide the results of the economic analysis of the current path(s). This synthesis should also describe the current financial and political commitments that support investments made by stakeholders in the current path, and discuss the implications for the current path if these change over time.

The investment case should discuss key uncertainties, limitations in the evidence, and other issues that may potentially impact the assessment of the current or status quo path(s), burden of disease, cost, and valuation estimates. The discussion should highlight any major issues that currently interfere with greater success of existing control efforts and identify the impacts of different assumptions on any quantitative results provided about the current path in appropriate sensitivity and/or uncertainty analyses.

Building on the foundation developed about the current situation (discussed in section 2), the investment case should identify the full set of potential options that exist for alternative levels of investment in global management of the disease from a decision point (i.e., a time zero or $\mathrm{T}_{0}$ for the change of path). Alternatives should explore different disease control targets (e.g. mortality reduction, eradication, and possibly regression or less control), different time lines for achieving those targets, and different timing, with full recognition of the potential for shared or competitive use of scarce resources. These should include appropriate details about the relevant expectations for individual countries and/ or regions at and after $\mathrm{T}_{0}$ for each alternative option (recognizing that nations and/or regions may start in different places given the variability in their current strategies discussed in 2.5). For any eradication alternative(s) the investment case should include a specific plan for achieving eradication with a proposed timeline, projections about how the world will look after eradication is achieved, and discussion of expected posteradication activities (e.g., expected needs for continued surveillance, intervention, intervention supply stockpiles, and/or outbreak response capabilities). The alternatives should also consider any potential add-on activities that may also lead to cost-sharing opportunities.

3.2 Global $\quad$ For each potential alternative (identified in 3.1) the investment case should determine whether sufficient supplies of interventions and diagnostic intervention supply tools exist to support implementation of the alternative and intervention supply security, particularly in the context of ongoing development of potentially more profitable products by intervention manufacturers. The information presented in this part may lead to iteration and adjustment of the options or reduction in the set of alternatives carried forward in the remainder of section 3 . The analysis should carefully consider the timelines required for any efforts to ramp up production to ensure that the alternative paths reflect real possibilities and to ensure consistency with estimates of resource requirements (discussed in 3.4). The estimates should also include the possibility of intervention supply disruptions and any reserves or stockpiles that may exist or need development. If outbreak response efforts for the disease(s) considered in the investment case or for other public health emergencies compete for intervention resources, then the investment case will need to discuss the management of this competition, and/ or if opportunities exist to share resources, then the investment case needs to characterize the potential benefits of sharing.

\begin{tabular}{l|l}
$\begin{array}{l}\text { 3.3 Global system } \\
\text { implementation }\end{array}$ & $\begin{array}{l}\text { The investment case should provide details about whether the existing systems (including surveillance and intervention delivery) exist to } \\
\text { support implementation of each alternative. The information in this part may lead to iteration and adjustment of the options (identified in } 3.1\end{array}$
\end{tabular} implementation capacity support implementation of each alternative. The information in this part may lead to iteration and adjustment of the options (identified in 3.1) or reduction in the set of alternatives carried forward throughout the remainder of section 3 . The analysis should carefully consider the timelines required for any efforts to build capacity to ensure that the alternative paths reflect real possibilities and to ensure consistency with estimates of resource requirements (discussed in 3.4). If creating and sustaining health systems capable of supporting delivery of the interventions represents an issue, then the interventions will need to identify strategies for addressing inadequacies (e.g., recruitment and training of dedicated staff, performing supplemental immunization activities) and ensure sufficient resources exist to support these activities (discussed in 4.2). If insufficient resources exist or cannot reasonably be anticipated to exist, then the alternative does not qualify as realistic and would not carry through to the remainder of section 3 . The investment case should provide a plan for ensuring no net harm to health systems associated with the alternative, and characterize any expected net benefits to health systems associated with implementation of the alternative. In addition if outbreak response efforts for this disease or for other public health emergencies compete for capacity, then the investment case will need to discuss the management of this competition.

\begin{tabular}{l|l}
3.4 Expected & For each of the feasible alternatives, the investment case will need to estimate the associated financial costs and its incremental cost
\end{tabular} financial costs of the compared to the current or status quo path(s). The analysis should use the same dollar year, discount rate, and assumptions to ensure fair comparisons with the costs of the current path(s) (discussed in 2.6). The expected costs should identify opportunities to share costs (i.e. potential synergies) between interventions and/or with other existing or potential efforts, but it should cost these separately such that the cost estimates do not depend on the occurrence of other actions (i.e., the cost estimates should reflect the resources required to pursue each alternative independently or together with clear linkage to and consistency with assumptions about timing). In addition, the investment case should include estimated global programmatic costs associated with each alternative, if implementation of the alternative requires an additional investment. For example, for an alternative that pursues eradication, the incremental global programmatic costs may need to include resources required to expand surveillance networks, social mobilization, laboratory and health worker resources, and volunteer capacity and/or to support communications (including social, anthropological, and behavioral) and operations research.

3.5 Expected net benefits of the alternatives The investment case should quantify the expected net health benefits of the alternatives using the same metrics and approaches used for the current path(s) (discussed in 2.7) and present the incremental net benefits for each alternative. The investment case should also identify potential positive and/or negative indirect impacts, including effects on health systems, for each option and quantify the impacts to the extent possible (with qualitative considerations discussed in section 4).

3.6 Expected political and financial commitments The investment case should identify the expected required political and financial commitments needed to support the various alternative paths, including critical factors for securing required commitments and global agreements. The discussion should also address how the alternatives in the investment case relate to other control and/or eradication initiatives in the global portfolio (discussed in part 1.4) and issues of competition and opportunities for synergies. This part should discuss the implications of potential changes in political, financial, and other resource commitments and investments, including consideration of the impact of delays of other global health initiatives leading to the potential of delayed or limited availability of resources.

3.7 Synthesis of the economics of the alternative investments
The investment case should synthesize overall evidence and provide an economic analysis of the expected alternatives compared to the current status quo path(s). This part should present estimates of incremental cost-effectiveness and net benefits for the alternatives compared to the current path(s) at the global level and, as needed, for individual countries and/or regions. For each alternative, the investment case should identify key milestones for tracking progress along the expected path. As needed, the investment case should present different metrics that may prove useful to different stakeholders and/or references for comparison (e.g., costs per capita compared to the health sector budget, costs of other initiatives). 
3.8 Limitations and The last part of the discussion of the alternatives should describe key uncertainties, limitations in the evidence, and other issues that may uncertainties $\quad$ potentially impact the assessment of the alternatives and the associated burden of disease or cost estimates. The discussion should highlight any major considerations (discussed in section 4 ) and provide the results of any sensitivity and/or uncertainty analyses performed. This part should demonstrate the impacts of explicit choices regarding valuation of health outcomes and nonmonetary benefits on the economic analyses.

4. Considerations

4.1 Broad social impacts

The investment case should discuss the differences in broader social impacts, including intergenerational benefits, equity (e.g., reaching marginalized populations), and social value for any investment case that includes alternative paths for which the social impacts differ from each other and/or the current path(s). If the investment case includes eradication as an alternative path it should consider the change in the nature of the public good and/or any social dilemmas that arise compared to the current path(s). This part should highlight any metrics of equity that might support discussion of the alternatives based on different criteria than the economics (e.g., percent of children reached through SIAs not otherwise currently reached). The discussion in this part should focus on significant differences related to the alternative paths compared to the current path(s), but also consider issues related to the societal effects of not meeting milestones for current commitments on the likelihood of support for future efforts, including the impacts of failing to deliver on prior promises recognized as overly optimistic with the passage of time. The investment case should consider the impacts of the current and alternative paths on social norms and expectations for health.

4.2 Impact on health The investment case should provide an assessment of why existing health systems stratified by relevant categories or types currently perform the way they do with respect to management of the infection(s) and disease(s) considered in the investment case. This analysis should identify the areas with the weakest, most fragile health systems and provide detailed plans for addressing the issues in these areas required to meet the targets associated with the current and alternative paths. The investment case should also identify a proposed process for active evaluation of any positive or negative impacts on health systems. The analysis of health systems should explicitly consider the sustainability of resources required to provide health services and infrastructure capacity, including overall cold chain demands and requirements and access to all areas (e.g., security, roads or other transport). The investment case should consider the potential impact of positively and/or negatively distorting the current incentives of stakeholders by introducing new resources (e.g., providing resources to build health worker capacity in weak health systems characterized by overloaded and stretched staff may inadvertently create dependency on external funds).

4.3 Management and The investment case should describe the management and organizational structures required for the current and alternative paths and governance describe the existing and expected roles and commitments of stakeholders for the current path and any differences in requirements for the alternative paths. The organizational structure should address all levels and their connections, and provide clarity about the management and governance of any activities coordinated globally and/or regionally. The investment case should also describe the expected tools and process for monitoring and evaluating progress toward milestones (discussed in 3.7) and responsibilities related to managing coordination, cooperation, and shared resources. In the context of an alternative path that includes eradication, this part should describe the specific organizational roles and responsibilities related to outbreak response, stockpile, surveillance, and other global programmatic activities. This part should address any issues of institutional capacity to provide programmatic support to engage stakeholders at all levels. For stakeholders that lack sufficient resources, the investment case should address the community level and/or national costs, perceptions, and/or incentives that may motivate and/ or discourage individuals, governments, and/or organizations from doing the work required to achieve the milestones.

4.4 Stakeholde engagement Achievement of milestones associated with the current and alternative paths will require the engagement of a broad group of stakeholders, including governments at all levels, donors, intervention manufacturers, civil society and non-governmental organizations (including professional societies, community groups, etc.), and individuals (who will need to accept and demand the interventions, perhaps on behalf of others, as in the case of parents making choices for their children). The investment case should provide details about plans for communication with stakeholders to engage them in the deliberative process to chart the preferred course and ensure alignment of their perceptions about the preferred course and incentives for them to support its implementation. To the extent that various stakeholders hold competing objectives, the investment case should make these clear and discuss potential opportunities for compromise and negotiation in addition to exploring the implications of the overall mixed messages that may arise. This part should include discussion of the expected roles and responsibilities of various stakeholders, and discuss the need for and the expected process for performing research related to understanding and managing perceptions related to the current and alternative paths. This should include an assessment of communication risks and discussion of expected communications efforts to engage stakeholders to address the risks identified (discussed in 4.5). The investment case should discuss the need for stakeholders to make and maintain commitments in the context of success, when the absence of cases of illness or disease may lead to misperceptions that individuals/countries/regions no longer need the intervention, and/or in the context of failures, when the resurgence of cases of illness or disease may undermine confidence and demonstrate the fragility of prior gains.

4.5 Risks and contingency plans

The investment case should clearly identify key risks and describe the detailed contingency plans that exist for their management. The investment case should anticipate challenges and constraints of all types (ethical, geopolitical, social, economic, epidemiologic, technical and institutional) for the alternatives that differ from the current path(s) and demonstrate plans and strategies to address these, including the allocation of resources to implement the contingency plans. In particular, this part should address issues related to maintaining progress toward the milestones in the context of financial crises, political strife or instability, and other emergencies. Given the reality that settings and situations differ (e.g., interventions and strategies that prove effective in some places may not perform as well in others), the investment case should consider the impact of uncertainties related to extrapolation to new conditions and provide context that will help stakeholders manage their expectations. The investment case should encourage the thorough investigation of the underlying causes of outbreaks, because outbreaks may represent failures of strategy, commitment, and/or implementation and global management efforts should apply the lessons learned from outbreaks to other areas with similar risks.

4.6 Research and The investment case should discuss the role of research with respect to resolving important sources of uncertainty and achieving the milestones development for the current and alternative paths. It should also identify knowledge gaps and research needs that will support the development of a process for supporting ongoing research, particularly operational and communications research, which may prove pivotal to success in some communities. The investment case should discuss the development of new tools that may lead to more cost-effective interventions. For significant basic research questions (e.g., pathogenesis, the mechanistic basis for the effectiveness of an intervention), the investment case should identify key questions and encourage the investigation of promising lines of research.

4.7 Timing in the The investment case should consider the broader context of other globally-coordinated disease management efforts and it should be viewed context of the portfolio an ongoing process. Major initiatives may in some cases benefit from synergies associated with shared use of infrastructure and/or other of globally-coordinated resources and in other cases they may compete for scarce resources (e.g., funding, political attention, health system and human resources, disease management public and media attention). The investment case should provide context about the overall portfolio of global disease management efforts efforts (discussed in part 1.3) and explore how the alternatives may benefit from synergies and/or may encounter additional challenges with respect to the timing of their implementation. This part should explore alternative timing options beyond those discussed in section 3 and address any important qualitative considerations (e.g., issues related to political will).

4.8 Financing The investment case should consider the opportunities that may exist for financing globally-coordinated efforts and how the investment case fit into the context of the overall portfolio of global disease management efforts (discussed in 1.3) and/or global health efforts more broadly to the extent possible. In the context of discussing potential financing, the investment case should focus on anticipated opportunities and challenges that may exist given other global commitments (e.g., building on the infrastructure of an existing eradication initiative, competing for a fixed poo of funds) and the potential impact on financing. 


\begin{abstract}
4.9 Oversight and iteration

\begin{tabular}{|l|l|}
\hline 5. Conclusions & $\begin{array}{l}\text { The investment case should provide a set of conclusions and include any recommendations about preferred alternatives with respect to any of } \\
\text { the attributes (i.e., it should identify the set of "best" options that exist based on the quantitative analyses and on specific qualitative factors). } \\
\text { The conclusions should reflect and support the deliberations by stakeholders (not preclude or preempt them) and make all assumptions clear } \\
\text { such that stakeholders can debate and iterate on the alternatives with the objective of reaching global consensus on and commitment to the } \\
\text { preferred path. }\end{array}$ \\
\hline End Matter & $\begin{array}{l}\text { The investment case should include a list of references, a list of acronyms, and a glossary that defines key terms and any technical appendices } \\
\text { needed to provide details about the data and models used for its quantitative analyses, which should provide enough detail that technical } \\
\text { readers could replicate the analyses. The technical appendices may also include details about the qualitative analyses of the health systems, } \\
\text { equity, or other issues. }\end{array}$
\end{tabular}

The investment case should discuss the planned process for oversight of global and regional coordination of efforts and consider the potential impact of time delays, unplanned activities (e.g., outbreak response), and programmatic cost overruns. It should also outline a process for updating the investment case, particularly as the current path(s) change(s) (e.g., if one of the alternatives becomes the new current or status quo path). Effective use of the investment case as a living document will require periodic iteration, and this will require an ongoing commitment to keep it updated. We anticipate that the process may need to include the creation of an independent oversight committee or monitoring board (i.e., separate from the organizations responsible for management discussed in 4.3).
\end{abstract}

Table 1: Content for an investment case for globally-coordinated management of infectious diseases.

investment cases might want to use this list as a starting point for iterations with their own appropriate set of stakeholders.

Our consultation process specifically asked about target audiences for investment cases. Overall, the stakeholders who responded identified a large number of target audiences for investment cases. Table 2 summarizes the complete list, which we categorized in to seven broad groups: Financial, public health policy makers, technical, health systems and intervention delivery, intervention producers, civil society, and lay audiences and the media. Many challenges in communication will arise in the context of developing investment cases that meet the needs of the various target audiences, and consequently we anticipate the need for multiple products that communicate the results.

Stakeholders also offered several notable comments related to the development of investment cases. First, some stakeholders raised the importance of maintaining objectivity in the development of the investment case so the cases present rigorous, balanced, pragmatic assessments of all options without bias or advocacy, realistic estimation of costs, and full characterization of risks. Second, they emphasized the importance of discussing past experience and potential impacts of options on existing programs, including routine immunization and other health services. With respect to past experience, stakeholders indicated the need to consider the future social costs of overly optimistic goals and the societal effect of not meeting current goals with respect to the willingness of key stakeholders to support future efforts. Given the programmatic and political uncertainty of meeting ambitious goals and the overall track record of existing disease elimination and eradication initiatives, the investment case should explicitly address the costs of failure. Third, stakeholders emphasized the challenges associated with heterogeneity and they identified countries with the weakest health systems, poorest overall infrastructure, least stable political and/or social environments, and fewest resources as the most challenging areas. With respect to these countries, they raised questions about both the optimal timing for investments and sustainability of efforts in these areas while also raising concerns about the widening gap between developed countries that continue to incorporate new strategies and tools, and some less-developed countries that continue to lose ground, political will, social capital, and critical infrastructure. The stakeholders also emphasized that regions, countries, and subpopulations will differ with respect to critical factors and this will necessitate investments in social mobilization and partnerships with members of civil society. Fourth, stakeholders emphasized the need for investment cases to clearly articulate the risks associated with lack of the desired commitments and actions by each of the stakeholders and risks associated with political strife, financial crises, and other emergencies. Fifth, stakeholders suggested the need for investment cases to discuss the role of research in developing new technologies and techniques, document trends in vaccine reluctance, refusal or politicization together, and demonstrate the effectiveness of strategies for implementing innovations and responding to challenges. Sixth, the stakeholders cautioned against over reliance on mathematical models and emphasized the need to explicitly consider uncertainties. They also highlighted the importance of assessing the feasibility and acceptability of the options. Finally, stakeholders recognized the role of manufacturers as key stakeholders and indicated the need for the investment case to consider issues related to vaccine security and supply chain management.

\section{Discussion}

The development of investment cases represents an important opportunity to shift public health management and planning toward the vision of GVAP [1]. Managing infectious diseases to prevent their occurrence requires a sustained commitment to investing in interventions that protect individuals [20]. For vaccine preventable diseases, this typically means investing sufficient resources in immunization to achieve and maintain high levels of population immunity, ideally at a level high enough to prevent transmission $[28,34]$. For other types of diseases (e.g., neglected tropical diseases), protecting individuals may require appropriately estimating DALYs

\begin{tabular}{|l|l|}
\hline Category & Stakeholders \\
\hline Financial & $\begin{array}{l}\text { Donors, funders, major partners/international partners (e.g., Bill \& Melinda Gates Foundation, GAVI Alliance, World Bank, OECD), } \\
\text { government development agencies (e.g., USAID, NORAD, DFID), Bi-laterals and Multi-laterals, broad financial community (private sector) }\end{array}$ \\
\hline Public health policy makers & $\begin{array}{l}\text { All levels of policy makers in the public health community, governments, (e.g., national Ministers of Health and Finance, national health } \\
\text { system and immunization officials, political leaders and elected representatives, bureaucrats and program managers) }\end{array}$ \\
\hline Technical & Scientific community, technical advisors, academics, national government staff, researchers \\
\hline Health systems & Pediatricians, clinicians, professional medical associations, nurses \\
\hline Intervention producers & Manufacturers, supply chain managers \\
\hline Civil society & $\begin{array}{l}\text { Religious, cultural, and opinion leaders, civil society organizations and non-governmental organizations, community mobilizers, educators, } \\
\text { special interest groups, volunteers }\end{array}$ \\
\hline Lay audiences & Media, interest groups, individuals \\
\hline
\end{tabular}

Table 2: Stakeholders identified as target audiences for investment cases. 
and valuing the benefits of effective interventions to benefit fully from economies of scale and scope [35], which we expect might occur in the context of investment cases.

Investment cases may significantly help countries and regions recognize the benefits of prevention, particularly in the context of avoiding unplanned and expensive emergency response efforts [34]. Investment cases should reveal the potential for shortages of necessary supplies and/or system capacity for prevention. For vaccine preventable diseases, inadequate global supplies of vaccine currently lead to difficult choices. If outbreak response activities use resources and vaccines that would otherwise go toward routine immunization or preventative supplemental immunization activities, then this could undermine the efforts of countries trying to pursue the desired path of prevention. If resources consistently go toward 'fire-fighting' then this could inadvertently create disincentives for countries to invest in prevention [36]. With national health leaders facing an increasingly complex set of choices [26-28], regional and global coordination will grow in importance, and we suggest the need for greater consideration of investment in vaccine stockpiles to better manage surges in demand that may feedback in undesirable ways [37].

Although investment cases cannot predict the future and stakeholders should not interpret their results as doing so, we believe that they can help public health leaders chart the best possible future given the resources available and put the available resources toward their best use. To the extent that resource limitations emerge as the explanation for preventable cases of disease and the associated health outcomes, we anticipate that the development of investment cases may help to expand the availability of resources for health interventions globally.

Uncertainties will continue to make planning difficult, but through the management and governance efforts described in investment cases we anticipate that coordination could improve performance and encourage appropriate investment in research that may help to resolve key uncertainties. In addition, significant variability that exists in the world with respect to exposure to and management of different diseases, and we expect this will continue to create the need for analyses that capture the situations, perspectives, and values of all individual countries and regions. We recognize that the time used as the starting point for a global initiative will influence the results of the economic analysis [10], and consequently investment cases will need to present economic results carefully and with appropriate context [15].

We anticipate that maintaining investments cases as "living documents" that evolve with time may help to ensure that they continue to support global coordination efforts and manage expectations about financial and other resource requirements. However, this iterative approach will also require an ongoing commitment by key stakeholders to regularly engage in an analytic and deliberative process to evaluate performance and options. Only time will tell whether investment cases will emerge as useful tools for management, and we suggest the need for open discussion of this new approach and process.

\section{Acknowledgment}

We acknowledge support for this work from the World Health Organization (WHO) under Contracts PO 200470477 APW. The contents of this article are solely the responsibility of the authors and do not represent the official views of the World Health Organization, which had no role in the study design, data collection, analysis, or interpretation, writing of the manuscript, or decision to submit the manuscrip for publication. We thank the following individuals for helpful information and/or discussions: Anindya Sekhar Bose, Casey Boudreau, Daniel Carter, Katie Cuming, Lisa Cairns, Thomas Cherian, Susan Chu, Stephen Cochi, Alya Dabbagh, Messere Eshetu, Andrea Gay, Tracey Goodman, Jim Goodson, Christopher Gregory, Mark
Grabowsky, L. Homero Hernandez, Edward Hoekstra, Joseph Icenogle, Suresh Jadavh, Sam Katz, Apoorva Mallya, Rebecca Martin, Balcha Masresha, Ali Jaffar Mohamed, Chris Morry, Walter A Orenstein, Mark Pallansch, Susan Reef, Kuotong Nongho Rogers (Tambie), Paul Rota, Emily Simons, Peter Strebel, Maya van den Ent, Maya Vijayaraghavan, Steve Wassilak, Wang Xiaojun, Laura Zimmerman, and anonymous respondents.

\section{References}

1. (2012) Global Vaccines Action Plan.

2. World Health Organization (2008) Guide for standardization of economic evaluations of immunization programmes, Geneva, Switzerland.

3. Barrett S (2003) Global disease eradication. J Eur Econ Assoc 1: 591-600

4. Thompson KM, Duintjer Tebbens RJ (2008) The case for cooperation in managing and maintaining the end of poliomyelitis: Stockpile needs and coordinated OPV cessation. Medscape J Med 10: 190

5. Cochi SL, Dowdle WR (2011) The eradication of infectious diseases: understanding the lessons and advancing experience. Chapter $1 \mathrm{In}$ : Cochi SL Dowdle WR (Eds.), Disease eradication in the 21st century: implications for global health. Cambridge, MA: MIT Press.

6. Aylward RB, Acharya A, England S, Agocs M, Linkins J (2003) Global health goals: lessons from the worldwide effort to eradicate poliomyelitis. Lancet 362 909-914.

7. World Health Organization (2001) Transmission of wild poliovirus type 2 apparent global interruption. Wkly Epidemiol Rec 76: 95-97.

8. Aylward RB (2011) Lessons from the late stages of the global polio eradication initiative. Chapter 2 In: Cochi SL, Dowdle WR (Eds.), Disease eradication in the 21st century: implications for global health. Cambridge, MA: MIT Press.

9. Thompson KM, Rabinovich R, Conteh L, Emerson Cl, Hall BF, et al. (2011) Group report: Developing an eradication investment case. Chapter $10 \mathrm{In}$ : Coch SL, Dowdle WR (Eds.), Disease eradication in the 21st century: implications for global health. Cambridge, MA: MIT Press, 133-148.

10. Thompson KM, Duintjer Tebbens RJ (2011) Challenges related to the economic evaluation of the direct and indirect benefits and the costs of disease elimination and eradication efforts. Chapter $9 \mathrm{In}$ : Cochi SL and Dowdle WR (eds), Disease Eradication in the 21st Century: Implications for Global Health. Cambridge, MA MIT Press, 115-130

11. GAVI Alliance (2004) Guidelines for Preparing Proposals for GAVI/Vaccine Fund Investment. Second draft, version 4, May 2004

12. World Health Organization (2005) Global Immunization Vision and Strategy (GIVS) 2006-2015

13. Ehreth J (2003) The global value of vaccination. Vaccine 21: 596-600.

14. Bloom DE, Canning D, Weston M (2005) The value of vaccination. World Econ 6: 15-39.

15. Thompson KM, Tebbens RJ (2007) Eradication versus control for poliomyelitis: An economic analysis. Lancet 369: 1363-1371.

16. Duintjer Tebbens RJ, Pallansch MA, Cochi SL, Wassilak SGF, Linkins J, et al (2011) Economic analysis of the global polio eradication initiative. Vaccine 29 334-343.

17. Nelson EA, Sack D, Wolfson L, Walker DG, Seng LF, et al. (2009) Financing children's vaccines. Vaccine 27: F12-F17.

18. Ozawa S, Stack ML, Bishai DM, Mirelman A, Friberg IK, et al. (2011) During the 'decade of vaccines,' the lives of 6.4 million children valued at $\$ 231$ billion could be saved. Health Aff 30: 1010-1020.

19. Stack ML, Ozawa S, Bishai DM, Mirelman A, Tam Y, et al. (2011) Estimated economic benefits during the 'decade of vaccines' include treatment savings gains in labor productivity. Health Aff 30: 1021-1028.

20. Wolfson LJ, Gasse F, Lee-Martin SP, Lydon P, Magan A, et al. (2008) Estimating the costs of achieving the WHO-UNICEF global immunization vision and strategy, 2006-2015. Bull World Health Organ 86: 27-39.

21. (2009) Global Polio Eradication Initiative strategic plan, 2010-2012.

22. WHO (2012) Global measles and rubella strategic plan, 2012-2020

23. WHO (2000) Maternal and Neonatal Tetanus Elimination by 2005. 
Citation: Thompson KM, Duintjer Tebbens RJD (2012) Development of Investment Cases for Globally-Coordinated Management of Infectious Diseases. J Vaccines Vaccin 3:164.doi:10.4172/2157-7560.1000164

24. Alum A, Rubino JR, ljaz MK (2010) The global war against intestinal parasitesshould we use a holistic approach? Int J Infect Dis 14: e732-e738.

25. Frothingham R, Stout JE, Hamilton CD (2005) Current issues in global tuberculosis control. Int J Infect Dis 9: 297-311.

26. Thompson KM, Dabbagh A, Strebel PM, Perry R, Gacic-Dobo M, et al. National and global options for managing the risks of measles and rubella (submitted).

27. Thompson KM, Duintjer Tebbens RJ (2012) Current polio global eradication and control policy options: Perspectives from modeling and prerequisites for OPV cessation. Expert Rev Vaccines 11: 449-459.

28. Thompson KM, Pallansch MA, Duintjer Tebbens RJ, Wassilak SGF, Kim JH et al. (2012) Pre-eradication vaccine policy options for poliovirus infection and disease control. Risk Anal In press.

29. World Health Organization (2010) Global eradication of measles: report by the secretariat. Sixty-third World Health Assembly, Geneva, Switzerland.

30. World Health Organization (2011) Proceedings of the global technical consultation to assess the feasibility of measles eradication, 28-30 July 2010. $\mathrm{J}$ Infect Dis 204: S4-S13.
31. WHO/UNICEF Joint Annual Measles Report (2009) Strengthening Immunization Services through Measles Control.

32. Measles outbreaks and progress towards meeting measles pre-elimination goals: WHO African Region, 2009-2010 (2011). Wkly Epidemiol Rec 86: 129 140

33. World Bank (1993) World Development Report: Investing in Health. Washington DC

34. Thompson KM, Pallansch MA, Duintjer Tebbens RJ, Wassilak SGF, Cochi SL (2012) Modeling population immunity to support efforts to end the transmission of live polioviruses. Risk Anal.

35. Conteh L, Engels T, Molyneux DH (2010) Socioeconomic aspects of neglected tropical diseases. Lancet 375: 239-247.

36. Thompson KM, Duintjer Tebbens RJ (2008) Using system dynamics to develop policies that matter: global management of poliomyelitis and beyond. System Dynamics Review 24: 433-449.

37. Thompson KM, Duintjer Tebbens RJ (2012) Optimal global vaccine stockpile design for endemic or epidemic vaccine-preventable diseases. 